\title{
Preoperative nutritional support in cancer patients with no clinical signs of malnutrition-prospective randomized controlled trial
}

\author{
Pawel Kabata • Tomasz Jastrzębski • Michał Kąkol • \\ Karolina Król • Maciej Bobowicz • Anna Kosowska • \\ Janusz Jaśkiewicz
}

Received: 1 May 2014 / Accepted: 21 July 2014 / Published online: 6 August 2014

(C) The Author(s) 2014. This article is published with open access at Springerlink.com

\begin{abstract}
Purpose Preoperative nutrition is beneficial for malnourished cancer patients. Yet, there is little evidence whether or not it should be given to nonmalnourished patients. The aim of this study was to assess the need to introduce preoperative nutritional support in patients without malnutrition at qualification for surgery.

Methods This was a prospective, two-arm, randomized, controlled, open-label study. Patients in interventional group received nutritional supplementation for 14 days before surgery, while control group kept on to their everyday diet. Each patient's nutritional status was assessed twice-at qualification (weight loss in 6 months, laboratory parameters: albumin, total protein, transferrin, and total lymphocyte count) and 1 day before surgery (change in body weight and laboratory
\end{abstract}

\footnotetext{
The protocol was registered in clinicaltrials.gov, study ID: NCT01894828

P. Kabata $(\bowtie) \cdot$ T. Jastrzębski • M. Kąkol • K. Król • M. Bobowicz • A. Kosowska $\cdot$ J. Jaśkiewicz

Department of Surgical Oncology, Medical University of Gdańsk, Smoluchowskiego 17, 80-952 Gdańsk, Poland

e-mail: pawel.kabata@gmail.com

T. Jastrzębski

e-mail: jasek@post.pl

M. Kakol

e-mail: mkakol311@gmail.com

K. Król

e-mail: karolinkapl@wp.pl

M. Bobowicz

e-mail: bobowicz@gmail.com

A. Kosowska

e-mail: akosowska@uck.gda.pl

J. Jaśkiewicz

e-mail: januszj@gumed.edu.pl
}

parameters). After surgery, all patients were followed up for 30 days for postoperative complications.

Results Fifty-four patients in interventional and 48 in control group were analyzed. In postoperative period, patients in control group suffered from significantly higher $(p<0.001)$ number of serious complications compared with patients receiving nutritional supplementation. Moreover, levels of all laboratory parameters declined significantly $(p<0.001)$ in these patients, while in interventional arm were stable (albumin and total protein) or raised (transferrin and total lymphocyte count).

Conclusions Preoperative nutritional support should be introduced for nonmalnourished patients as it helps to maintain proper nutritional status and reduce number and severity of postoperative complications compared with patients without such support.

Keywords Cancer-related malnutrition · Preoperative nutrition $\cdot$ Nutritional supplementation $\cdot$ Cancer surgery

\author{
Abbreviations \\ ESPEN European Society for Clinical Nutrition and \\ Metabolism \\ TLC Total lymphocyte count \\ ERAS Enhanced recovery after surgery \\ CTCAE Common Terminology Criteria for Adverse Events
}

\section{Introduction}

Gastrointestinal cancer patients are at high risk for diseaserelated malnutrition and cachexia. This is a result of many coexisting factors including aggressive and catabolic biology 
of the disease, food intake and intestinal passage disturbances, and stress-related anorexia [1]. Therefore, metabolic deterioration of these patients starts long before its clinical effects can be seen.

As far as malnourished gastrointestinal cancer patients are concerned, numerous randomized trials and guidelines on preoperative nutrition were published [2-8]. All of these agree that preoperative nutrition is beneficial and necessary for these patients, as it improves their metabolic status and results in better postoperative outcomes, especially in means of complications. According to European Society for Clinical Nutrition and Metabolism (ESPEN) guidelines, supplementation should be given for 10-14 days preoperatively, even if surgery must be delayed [3-5]. At the same time, there is very little evidence on preoperative nutrition of nonmalnourished cancer patients. Most of currently available information comes from single randomized controlled trials (RCTs), or as secondary results of malnutrition trials, with incoherent results $[6,9]$. The question, whether or not preoperative nutrition should be introduced in these patients is very important, as this group is highly represented, especially among less aggressive neoplasms, e.g., colorectal cancer. Even though these patients do not meet the criteria for malnutrition (albumin level, $<35 \mathrm{~g} / \mathrm{l}$; weight loss, $>10 \%$; body mass index (BMI), <18.5) [10], their metabolic status is often already affected by the diseaserelated catabolism, which if uncontrolled, can lead to malnutrition.

To answer this question, we set up a trial to determine whether preoperative nutritional support should be routinely used in gastrointestinal cancer patients with no signs of malnutrition, and if such approach will have positive clinical effects.

\section{Materials and methods}

The study was a prospective single-center, two-arm, randomized, controlled, open-label trial. All patients qualified by the study team for elective, radical gastrointestinal, and abdominal cancer surgery in the Department of Surgical Oncology of Medical University of Gdansk, Poland between May 2011 and April 2013 were screened for eligibility. Screening was based on patients' medical data and clinical and radiological findings.

To enter the study, patients had to meet all inclusion criteria. All patients were adults in good general condition (>70 in Karnofsky scale) [11] with either primary or secondary gastrointestinal and abdominal cavity malignancy, not meeting criteria for malnutrition (albumin level, $<35 \mathrm{~g} / \mathrm{l}$; weight loss, $>10 \%$ ) at screening. The tumor had to be resectable based on preoperative radiological and clinical examinations at the moment of qualification for surgery. Patients with other major gastrointestinal diseases or signs of mechanical ileus were not eligible to participate. Also, other serious medical conditions, which may affect nutritional status, as well as renal and liver failure excluded from the study.

Eligible patients were randomized to one of two parallel study groups - interventional or control. Randomization was based on a computer-generated simple randomization table prepared by an independent statistician. No blocking or stratification was used. Patients in interventional arm were given on-market oral nutritional supplements (Nutridrink Protein ${ }^{\circledR}$, N. V. Nutricia) in addition to their normal diet. They were asked to drink two 200-ml bottles of this hypercaloric formula $(1.5 \mathrm{kcal} / \mathrm{ml})$ daily for 14 days before surgery. Each bottle contained $20 \mathrm{~g}$ of protein. Patients in the control group were asked to keep on to their normal eating habits. No supplementations or changes in composition of their diet were made.

All patients were checked for signs of malnutrition twice. First assessment was performed at qualification for the study and second, after hospital admission, 1 day before the surgery. The awaiting period for surgery was longer than intervention time (median 19 days in interventional and 18 in control group, with no statistical significance); therefore, patients were asked to start drinking nutritional supplements exactly 14 days prior to scheduled hospital admission. Initial screening comprised patients' age and general performance (Karnofsky scale), current weight, BMI, and weight loss within 6 months before qualification for surgery. Blood samples for laboratory tests - albumin, total protein, transferrin level, and total lymphocyte count (TLC) were obtained. Weight loss assessment was based on patients' information on body weight 6 months earlier and weight check at qualification for the study. At hospital admission, patients were checked for change in the body weight, BMI, and blood parameters' values since qualification for the study. Perioperative management of all patients was performed according to Enhanced Recovery After Surgery (ERAS) protocols. After surgery, depending on type of procedure performed, patients were given immediate nutritional intervention. Patients after oesophageal and gastric resections received enteral nutrition for 4-5 days followed by introduction of oral feeding. Rest of the patients was treated with oral nutrition supplemented by ONS, if necessary starting from first day after surgery.

After surgery all patients were followed-up for postoperative complications for 30 days. Complications' occurrence and their severity according to Common Terminology for Criteria for Adverse Events (CTCAE) classification were noted [12]. This is a five-grade classification, where: grade 1 stands for mild complication requiring no intervention, grade 2-moderate, when minor intervention is indicated. Grade 3 complications require intervention including pharmacological, surgical, and hospital admission if necessary, grade 4 are life-threatening complications requiring intensive treatment including immediate surgery and grade 5 stands for death. For the purpose of this study, severity and types of postoperative complications were 
grouped. Complications in grades 1-2 were treated as mild, and $3-5$ as severe. We also divided them in three main typesinfectious, surgical, and general.

Local ethics committee approval was obtained and all patients signed informed consent. The study protocol was registered in clinicaltrials.gov database, study ID: NCT01894828

\section{Statistics}

All data were gathered and analyzed on Per Protocol basis using IBM SPSS 20.0 software. Normality was assessed using the Kolmogorov-Smirnov test. Statistical analyses of data within patient groups were performed using the Wilcoxon signed ranks test. Mann-Whitney $U$ test was used to compare change in values between the groups. Fisher's exact test was used to compare discrete variables. $p \leq 0.05$ was considered to be statistically significant. Data are presented as median value with first (Q1) and third quartile (Q3) or number of patients.

Complications incidence and severity were analyzed between the groups. We assumed an overall complication rate for $30 \%$ in patients not receiving nutritional supplementation. Reduction to the level of $15 \%$ was considered proving efficacy of nutritional supplementation.

Sample size was calculated at $\mathrm{CI}=95 \%$ and $80 \%$ power for 200 patients in each arm. During the study, based on clinical observations of both groups, we noticed higher occurrence of complications in control group, therefore we decided to perform an interim analysis. It revealed significantly higher number of serious postoperative complications in control group compared with those receiving supplementation. There were also laboratory signs of metabolic deterioration of these patients.

\section{Results}

From May 2011 to April 2013, 113 patients entered the study. Four patients were preoperatively disqualified from surgical treatment after entering the study, and two patients did not show up for hospital admission. The four disqualified patients were of borderline resectability, later considered unresectable after preoperative reevaluation of diagnostic images at a reference radiology department. Five patients had incomplete data. They were not included in the final analysis, because it was impossible to calculate changes in laboratory parameters' and body weight values without all results. One hundred two patients with complete data were analyzed-54 in interventional and 48 in control group. Interventional group comprised of 24 male and 30 female patients; 28 men and 20 women were included in control group. Detailed information on disease characteristics are gathered in Table 1.
Baseline values of anthropometric parameters at qualification for the study were comparable between the groups (Table 2). However median age and body weight were higher in control group (67 vs 60 years and $74 \mathrm{vs} 68 \mathrm{~kg}$, respectively), while median percent weight loss was higher in interventional group (7.4 vs $6.3 \%$ ). These differences were not significant. Also, laboratory parameters values were similar in both groups, with no significant differences. At this moment, patients in both groups did not meet criteria for malnutrition. What is more, high BMI values (24.2 vs 26.1$)$ were at borderline for overweight. Yet, it is worth noticing that 6-month weight loss around $7 \%$ and albumin level in lower reference values may indicate already existing disease-related catabolism in both study groups.

On second assessment, changes in laboratory parameters and body weight values were measured. Significant differences $(p<0.001)$ between study groups were observed regarding all laboratory parameters' values and body weight. Values of all measured parameters decreased significantly $(p<0.001)$ in control group, with albumin and weight loss values at borderline level for malnutrition, whereas in supplementation group their levels increased. Significant changes were observed in median body weight $(p<0.001)$, transferrin $(p=0.032)$, and TLC $(p=$ 0.05 ) values. Albumin and total protein levels changed slightly, with no statistical significance (Table 2).

\section{Postoperative complications}

Number and severity of postoperative complications differed between study groups (Tables 3 and 4). Total number of complications was significantly higher in control group (17 vs $8 ; p=$ $0.04)$. These differences were also visible when divided into subgroups, by their severity — mild (6 vs $3 ; p=0.036$ ) and severe (11 vs $5 ; p<0.001)$. The assumed reduction of total complications' percentage in patients receiving nutritional supplementation compared with control group was achieved (from 35.4 to $14.8 \%$ ). Patients in control group suffered from higher number of infectious complications, mainly wound infections. However, pneumonia and sepsis, one case of each, were observed only in patients receiving supplementation. Although surgical complications occurred in both groups comparably, those, which are strictly connected with nutritional status - anastomotic leakage and evisceration, were observed only in patients without supplementation. Severe general complications, including one fatal, being a result of mixed cardiac-neurologic incident, were also observed only in control group.

\section{Discussion}

Preoperative nutrition plays an important role in preoperative management of surgical patients. It helps to restore proper 
Table 1 Types and numbers of cancers among patients in both study groups

\begin{tabular}{lll}
\hline & $\begin{array}{l}\text { Interventional } \\
\text { arm }(N=54)\end{array}$ & $\begin{array}{l}\text { Control arm } \\
(N=48)\end{array}$ \\
\hline Stomach & 11 & 9 \\
Rectum & 10 & 15 \\
Sigmoid colon & 5 & 9 \\
Ascending colon & 5 & 4 \\
Ovaries & 5 & 0 \\
Secondary abdominal tumor & 4 & 2 \\
Descending colon & 3 & 2 \\
Esophagus & 3 & 2 \\
Primary abdominal tumor & 3 & 1 \\
Peritoneal pseudomyxoma & 1 & 1 \\
Anus & 1 & 1 \\
Pancreas & 1 & 0 \\
Transverse colon & 1 & 0 \\
Appendix & 1 & 0 \\
Liver & 0 & 1 \\
Small intestine & 0 & 1 \\
\hline
\end{tabular}

nutritional status, which results in reduced number of postoperative complications. According to international guidelines, it should be given for 10-14 days before surgery. However, these guidelines generally apply to malnourished patients, which require intensive nutritional support with specialized diets, often in hospital setting.

According to the ESPEN guidelines, weight loss $>10 \%$ in 6 months is a risk factor for postoperative complications, and is borderline value for malnutrition. Even though this factor has been broadly studied, literature data on what level should be treated as threatening are inconsistent. Makela et al. in a case control study on a group of 44 left-sided colon cancer patients found statistically higher incidence of anastomotic leakage in subjects with weight loss $>5 \%$, which was also proven by Gregg to be a negative predicting factor for early postoperative death in patients with bladder cancer [13, 14]. On the other hand, Antoun et al. found higher incidence of severe postoperative complications in patients with over $15 \%$ weight loss [15]. Patients in our study at screening suffered from median weight loss at the level of 6-7 \%, which already had a negative impact on postoperative outcome. Having in mind that cancer-related weight loss and deterioration is a longterm and gradual process, basing on the results of our and other studies we think, that oral supplementation should be given to any cancer patient who suffered weight loss before surgery. It is also of utmost importance to screen each of these patients for malnutrition and nutritional risk, not relying only on weight loss values. The available screening tools can provide us additional and necessary information on signs and symptoms of the disease, which helps to make decision on what approach would be best for the patient.
Table 2 Values of demographic and laboratory parameters of patients at qualification for surgery and in second assessment, after the intervention/control (median values)

\begin{tabular}{|c|c|c|c|c|c|c|c|c|}
\hline & \multicolumn{4}{|c|}{ Interventional $\operatorname{arm}(N=54)$} & \multicolumn{4}{|c|}{ Control arm $(N=48)$} \\
\hline & Median & Q1 & Q3 & $p$ value & Median & Q1 & Q3 & $p$ value \\
\hline \multicolumn{9}{|l|}{ Demographic (baseline values) } \\
\hline Age & 60 & 49 & 68 & & 67 & 61 & 73 & \\
\hline Height (m) & 1.68 & 1.63 & 1.75 & & 1.68 & 1.64 & 1.75 & \\
\hline Body weight (kg) & 68 & 58 & 82 & & 74 & 63 & 86 & \\
\hline BMI $\left(\mathrm{kg} / \mathrm{m}^{2}\right)$ & 24.2 & 21.2 & 28.7 & & 26.1 & 22.5 & 28.4 & \\
\hline Weight loss in 6 months $(\mathrm{kg})$ & -5 & -10 & -1 & & -5 & -8 & -1 & \\
\hline$\%$ weight loss in 6 months & 7.4 & 9.5 & 0.6 & & 6.3 & 9.6 & 1.8 & \\
\hline Karnofsky score & 80 & 80 & 90 & & 80 & 80 & 90 & \\
\hline \multicolumn{9}{|l|}{ Nutritional (baseline values) } \\
\hline Albumin (g/l) & 38 & 36 & 41 & & 38 & 36 & 42 & \\
\hline Total protein $(\mathrm{g} / \mathrm{l})$ & 70 & 67 & 74 & & 71 & 67 & 75 & \\
\hline Transferrin $(\mathrm{g} / \mathrm{l})$ & 2.44 & 2.20 & 2.84 & & 2.50 & 2.16 & 2.89 & \\
\hline Total lymphocyte count (G/l) & 1.53 & 1.13 & 2.08 & & 1.76 & 1.23 & 2.08 & \\
\hline \multicolumn{9}{|c|}{ Nutritional (after intervention/control) } \\
\hline Body weight (kg) & 70 & 59 & 84 & $<0.001$ & 73 & 61 & 86 & $<0.001$ \\
\hline Albumin (g/l) & 39 & 35 & 43 & 0.849 & 34 & 31 & 41 & $<0.001$ \\
\hline Total protein $(\mathrm{g} / \mathrm{l})$ & 72 & 66 & 78 & 0.075 & 67 & 59 & 75 & $<0.001$ \\
\hline Transferrin $(\mathrm{g} / \mathrm{l})$ & 2.51 & 2.24 & 3.04 & 0.032 & 2.19 & 1.66 & 2.86 & $<0.001$ \\
\hline Total lymphocyte count (G/l) & 1.65 & 1.03 & 2.38 & 0.05 & 1.38 & 0.7 & 1.98 & $<0.001$ \\
\hline
\end{tabular}


Table 3 Incidence of postoperative complications in both study groups

\begin{tabular}{lll}
\hline Complication & $\begin{array}{l}\text { Interventional } \\
\operatorname{arm}(N=54)\end{array}$ & $\begin{array}{l}\text { Control arm } \\
(N=48)\end{array}$ \\
\hline Infectious & & 7 \\
$\quad$ Wound infection & 1 & 0 \\
Pneumonia & 1 & 0 \\
$\quad$ Sepsis & 1 & \\
Surgical & & 2 \\
Subileus & 2 & 1 \\
Mechanical ileus & 1 & 0 \\
Gastric bleeding & 1 & 0 \\
Esophageal graft perforation & 1 & 2 \\
Anastomotic leakage & 0 & 2 \\
Evisceration & 0 & \\
Other general & & 1 \\
Fluid-electrolyte disturbances & 0 & 1 \\
Heart attack & 0 & 17 \\
Death & 0 & 8
\end{tabular}

In literature, there is little evidence on what approach should be taken towards patients without malnutrition, and the data available are inconclusive. There have been several studies performed on this group of surgical patients, but the differences in their methodology and equivocal results render comparisons very difficult $[16,17]$. Most of these trials compared enteral and parenteral nutrition, with control groups not receiving any support. The nutrition period was 5-7 days, and all of the authors used immuno-enhanced formulas. Even though, the clinical effect of these studies, seen in reduced number of complications was similar to ours, it is hard to compare them with our study. Not only did we use oral formulas without immuno-enhancement but also time of the intervention was longer. To our best knowledge, there has been only one study, which concentrated on preoperative oral nutritional supplementation, however again with immunoenhancement [18]. Giger-Pabst et al. in a RCT- administered

Table 4 Severity of postoperative complications according to CTCAE classifications

\begin{tabular}{llll}
\hline Complication & $\begin{array}{l}\text { Interventional } \\
\operatorname{arm}(N=54)\end{array}$ & $\begin{array}{l}\text { Control arm } \\
(N=48)\end{array}$ & $p$ value \\
\hline Grade 1 (mild) & 2 & 5 & 0.036 \\
Grade 2 (moderate) & 1 & 1 & $<0.001$ \\
Grade 3 (severe) & 4 & 6 & \\
Grade 4 (life threatening) & 1 & 4 & \\
Grade 5 (death) & 0 & 1 & \\
Total & 8 & 17 & \\
\hline
\end{tabular}

oral formulas for 3 days preoperatively, but with no differences in clinical outcome between the groups. In our opinion, this was caused by relatively short time of treatment, which despite stimulation of the immune system, was not sufficient to exert expected biological response. Prolonging the intervention time in our study enabled reduction of postoperative complications and stopped patients' catabolic deterioration, seen in stable levels of laboratory parameters. The 14-day period of supplementation seems to be the shortest necessary to achieve such effect. It is possible to prolong this period with oral nutritional supplementation, because unlike enteral or parenteral formulas, it does not require hospital setting and can be used at home in the awaiting presurgical period.

Oral nutritional supplements are generally very well tolerated, which results in high compliance. Their consistency, together with high-energy load and small volume needed to provide sufficient amount of nutritional components enable proper nutrition even for patients with dysphagia or intestinal passage disturbances. Therefore, they are commonly used in oncological and geriatric patients who experience this type of difficulties. Although many studies regarding compliance to ONS have been published so far, to our best knowledge only one systematic review has been made to summarize those studies [19]. Hubbard et al. reviewed 46 studies of all types with 4,328 patients in which compliance to ONS was measured. Those studies compared patients with various diseases (oncological, geriatric, respiratory, renal, and fractures) receiving supplementation in ambulatory as well as inpatient setting. The overall compliance to prescribed ONS was $78 \%$ with most of studies $(62 \%)$ at the level of $>75 \%$. This high compliance was seen regardless of patient type, disease characteristics or healthcare setting. Even though in our study compliance to ONS was not calculated separately, it has been checked based on verbal response of our patients. We did not notice any significant noncompliance to the prescribed ONS, which corresponds with the results of cited studies.

Few authors questioned superiority of immunomodulating formulas in preoperative nutrition compared with formulas not containing these agents. In numerous studies, it has been shown that there were no differences in overall mortality, morbidity, and cost-effectiveness between these approaches [8, 20]. Some of them pointed out that timing of the nutrition is more important factor than its type. Klek et al. in a randomized trial comparing different approaches towards perioperative nutrition showed that regardless of nutrition content, preoperative administration is of utmost importance to prevent postoperative complications. [21] Even though in our study the supplements used were without immunostimulating agents, the biological effect achieved was similar to those, which used enteral immunoenriched formulas. The number of infectious complications, mainly surgical site infections, was reduced, which was a result of positive impact exerted on immunological system by the supplements. Yet, these complications, although most frequent, are not life 
threatening, and less significant from clinical point of view. With nutritional supplementation, we managed to reduce number of strictly surgical complications connected directly with one's nutritional status, such as anastomotic leakage or evisceration.

Higher incidence of severe complications, observed in course of the study was the reason for performing a, previously not-planned interim analysis. Even though the number of patients included did not reach the calculated one, its results were sufficient to draw conclusions. Therefore, we decided to terminate the study prematurely.

\section{Conclusions}

Preoperative nutritional support is beneficial and should be routinely used in abdominal and gastrointestinal cancer patients with no clinical signs of malnutrition. Such approach reduces the number and severity of postoperative complications, especially of anastomotic dehiscence and leakage. Oral nutritional supplementation with no immunomodulation given for 14 days divides the total number of postoperative complications in patients with gastrointestinal cancers.

Statement of authorship PK, MK, TJ, and JJ designed the study. PK, $\mathrm{MK}, \mathrm{KK}$, and AK gathered the data and performed study related procedures. PK and TJ analyzed and interpreted the results. PK, MB, and TJ prepared and reviewed the manuscript. All authors read and approved the final manuscript.

\section{Sources of funding Internal funding}

\section{Conflict of interest None}

Open Access This article is distributed under the terms of the Creative Commons Attribution Noncommercial License which permits any noncommercial use, distribution, and reproduction in any medium, provided the original author(s) and the source are credited.

\section{References}

1. Argiles J (2005) Cancer-associated malnutrition. Eur J Oncol Nurs 9: 39-50

2. Bozzetti F, Braga M, Gianotti L, Gavazzi C, Mariani L (2001) Postoperative enteral versus parenteral nutrition in malnourished patients with gastrointestinal cancer: a randomized multicentre trial. Lancet 358:1487-1492

3. Bozzetti F (2010) Basics in clinical nutrition: nutritional support in cancer. Eur J Clin Nutr Metab 5:148-152

4. Bozzetti F (2001) Nutrition and gastrointestinal cancer. Curr Opin Clin Nutr Metab Care 4:541-546
5. Ljungqvist O, Dardai E, Allison S (2010) Basics in clinical nutrition: perioperative nutrition. Eur J Clin Nutr Metab 5:93-96

6. Braga M, Gianotti L, Nespoli L, Radaelli G, Di Carlo V (2002) Nutritional approach in malnourished surgical patients. Arch Surg 137:174-180

7. Braunschweig C, Levy P, Sheean P, Wang X (2001) Enteral compared with parenteral nutrition: a meta-analysis. Am J Clin Nutr 74: 534-542

8. Burden S, Todd C, Hill J, Lal S (2012) Pre-operative nutrition support in patients undergoing gastrointestinal surgery. (Review). Cochrane Database Syst Rev 11:1-64

9. Jie B, Jiang Z, Nolan M, Efron D, Zhu S, Yu K, Kondrup J (2010) Impact of nutritional support on clinical outcome in patients at nutritional risk: a multicenter, prospective, cohort study in Baltimore and Beijing teaching hospitals. Nutrition 26:1088-1093

10. Nespoli L, Coppola S, Gianotti L (2012) The role of enteral route and the composition of feeds in the nutritional support of malnourished surgical patients. Nutrients 4:1230-1236

11. Karnofsky D, Abelmann W, Craver L, Burchenal J (1948) The use of nitrogen mustard in the palliative treament of cancer. Cancer 22:634 656

12. Common Terminology Criteria for Adverse Events version 4.03 (2010) National Institute of Health

13. Makela J, Kiviniemi H, Laitinen S (2003) Risk factors for anastomotic leakage after left-sided colorectal resection with rectal anastomosis. Dis Colon Rectum 46:653-660

14. Gregg J, Cookson M, Philips S, Salem S, Chang S, Clark P, Davis R, Stimson C Jr, Aghazadeh M, Smith J Jr, Barocas D (2011) Effect of pre-operative nutritional deficiency on mortality after radical cystectomy for bladder cancer. J Urol 185:90-96

15. Antoun S, Rey A, Beal J, Montagne F, Pressoir M, Vasson M, Dupoiron D, Gourdiat-Borye A, Guillaume A, Maget B, Nitenberg G, Raynard B, Bachmann P (2009) Nutritional risk factors in planned oncologic surgery: what clinical and biological parameters should be routinely used? World J Surg 33:1633-1640

16. Gianotti L, Braga M, Nespoli L, Radaelli G, Beneduce A, Di Carlo V (2002) A randomized controlled trial of preoperative oral supplementation with a specialized diet in patients with gastrointestinal cancer. Gastroenterology 122:1763-1770

17. Horie H, Okada M, Kojima M, Nagai H (2006) Favourable effects of preoperative enteral immunonutrition on a surgical site infection in patients with colorectal cancer without malnutrition. Surg Today 36 : 1063-1068

18. Giger-Pabst U, Lange J, Maurer C, Bucher C, Schreiber V, Schlumpf R, Kocher T, Schweizer W, Krahenbuhl S, Krahenbuhl L (2013) Short-term preoperative supplementation of an immunoenriched diet does not improve clinical outcome in well-nourished patients undergoing abdominal cancer surgery. Nutrition 29:724-729

19. Hubbard G, Elia M, Holdoway A, Stratton R (2012) A systematic review of compliance to oral nutritional supplements. Clin Nutr 31: 293-312

20. Sodergren M, Jethwa P, Kumar S, Duncan H, Johns T, Pearce C (2010) Immunonutrition in patients undergoing major upper gastrointestinal surgery: a prospective double-blind randomised controlled study. Scand J Surg 99:153-161

21. Kłęk S, Sierzega M, Szybinski P, Szczepanek K, Scislo L, Walewska E, Kulig J (2011) Perioperative nutrition in malnourished surgical cancer patients - a prospective randomized, controlled clinical trial. Clin Nutr 30:708-713 